\title{
Philosophiques
}

Gilles Lane. Si les marionnettes pouvaient choisir. Recherche sur les droits, l'obligation morale et les valeurs. Montréal, L'Hexagone, coll. Positions Philosophiques, 1983, 211 p.

\section{André Carrier}

Volume 12, numéro 2, automne 1985

URI : https://id.erudit.org/iderudit/203297ar

DOI : https://doi.org/10.7202/203297ar

Aller au sommaire du numéro

Éditeur(s)

Société de philosophie du Québec

ISSN

0316-2923 (imprimé)

1492-1391 (numérique)

Découvrir la revue

Citer ce compte rendu

Carrier, A. (1985). Compte rendu de [Gilles Lane. Si les marionnettes pouvaient choisir. Recherche sur les droits, l'obligation morale et les valeurs. Montréal,

L'Hexagone, coll. Positions Philosophiques, 1983, 211 p.] Philosophiques, 12(2),

451-454. https://doi.org/10.7202/203297ar d'utilisation que vous pouvez consulter en ligne.

https://apropos.erudit.org/fr/usagers/politique-dutilisation/ 
GILLES LANE. Si les marionnettes pouvaient choisir. Recherche sur les droits, l'obligation morale et les valeurs. Montréal, L'Hexagone, coll. Positions Philosophiques, 1983, $211 \mathrm{p}$.

\section{par André Carrier}

Partant de l'examen des croyances et des comportements impliqués dans la revendication des droits, Gilles Lane entend concevoir les conditions de possibilité de la liberté. Il démontre qu'elles sont les mêmes que celles ayant trait à la revendication des droits et à l'exercice des obligations dites morales.

Le chapitre premier porte sur l'analyse de ce qui se passe lorsqu'il y a revendication d'un droit : l'homme, aspirant à conduire de lui-même sa propre vie, se reconnaît des droits, souhaitant ainsi modifier le comportement d'autrui ; il attend de l'autre qu'il s'oblige lui-même, ce qui caractérise la pression morale (à la différence de toute force ou contrainte et de toute pression psychologique) ; il compte sur le fait que cet autre soit tenu, qu'il reconnaisse, par simple constatation, l'exigence d'une obligation à laquelle il doit de lui-même consentir, et ce en dépit de ses désirs ou de ses projets factuels ; pour être morale, l'obligation devra cependant ne pas être aveugle.

Lane nous démontre par la suite que l'enjeu fondamental des droits n'est pas tel ou tel comportement exigible, mais " la possibilité pour chacun de vivre en plus grande confiance parmi ses « semblables », et plus précisément, de poursuivre avec une confiance toujours mieux fondée, et mieux protégée, 
la satisfaction ou la réalisation de certains besoins, désirs, ou aspirations » (p. 29). Quant au fondement de cette confiance et quant à la possibilité d'un recours lors de la revendication des droits, il semble qu'ils doivent reposer ailleurs que sur la promesse ou que sur la promesse de tenir ses promesses ; face à ces contrats plutôt aléatoires, il doit en effet y avoir "l'équivalent d'un engagement qui serait cette fois irrémédiable en lui-même » (p. 36).

Cet engagement, au fondement de la confiance, consiste à maintenir une attitude favorable à l'endroit de l'autre. Il suppose pourtant la liberté de jugement et d'approbation à l'égard de ses projets. Ceci aurait d'ailleurs pour effet de susciter chez l'autre une crainte ou un doute, s'il n'était préalablement assuré, non pas de réaliser tel ou tel projet, mais de bénéficier d'une attitude bienveillante à son égard. Décidé, l'engagement à être bienveillant devient moralement irrémédiable. Et la raison de cet engagement se trouve par l'examen des conditions de possibilité du libre choix. Cellesci apparaissent aux chapitres IV et $\mathrm{V}$ sous l'angle du faire, du vouloir faire, de la rencontre de l'autre et de l'invitation.

Malgré l'impossibilité apparente de la liberté due aux hiérarchies de valeurs préexistantes dans lesquelles il puise, l'homme persiste à croire qu'il n'agit pas n'importe comment, ni au hasard, mais de manière à réaliser une valeur qui ne soit pas non plus n'importe laquelle. Il pose d'ailleurs à son sujet la question du pourquoi, laquelle cesse de rebondir indéfiniment lorsque la chose à faire se révèle ou est perçue comme valant pour elle-même. Quelle est cette chose qui vaut en elle-même ? Il s'agit, comme nous n'agissons jamais seuls, d'atteindre l'autre pour lui-même. Le raisonnement de Lane est le suivant : faire, c'est vouloir faire ; c'est donc attendre la venue de ce que l'on veut faire, et faire en sorte que la chose advienne, en suscitant l'équivalent d'une réponse à notre attente. Vouloir faire est donc l'équivalent d'attendre d'un autre qu'il détermine la venue des choses que l'on veut faire. Il s'agit d'une invitation portée à cet autre ; il s'agit d'une contribution et d'une volonté réciproques entre semblables de s'atteindre pour soi-même. Faire de soi-même est donc faire avec un autre au cœur des rapports humains où tous, visant la rencontre, se donnent interminablement leur attention. La possibilité de l'activité libre est donc attribuable à la capacité de l'homme d'agir de lui-même et d'être le seul et unique point de départ immédiat de vouloirs conscients. Il s'agit d'une capacité d'être "source inépuisable " ou « jaillissement interminable " du don tout à fait originaire d'une attention portée à un autre pour lui-même " (p. 87). Cette source d'attention pour autrui serait inaliénable. Elle demeure libre car toujours "à venir ", " capable d'aimer, d'être aimable et d'être aimée ". Cette conception implique " qu'aucun être, y compris un Dieu, ne pourrait agir de lui-même (. . .) s'il ne visait, par son activité, à atteindre un autre point de départ d'activités propres, pour faire l'équivalent de lui donner, si cet autre voulait bien l'accepter de lui-même, une « attention » tirée de lui-même seul, et simplement pour en atteindre cet autre en ce qu'il est lui-même, interminablement » (p. 88).

Invité à la rencontre, l'autre ne l'accepte que s'il le veut bien. Il peut toujours refuser, malgré le caractère obligatoire de cette invitation libératrice, 
de cette interminable « rencontre d'appréciation mutuelle ». Obligé, l'autre demeure libre, n'échappant pas davantage à sa liberté qu'à l'obligation morale.

Avant de procéder à un examen des implications sociales de sa théorie (chap. VII), Gilles Lane analyse les incidences de celle-ci sur les valeurs (chap. VI), questionnant ce qui est bon ou mauvais. Leur discrimination est condition de liberté, et se pose dans le contexte d'une intervention à produire, d'où la notion de "préférabilité objective " d'un choix ou d'une décision. Pour ne pas valoriser n'importe quoi, il faut que la chose mérite préalablement d'être valorisée et soit telle « qu'un être peut vouloir de lui-même la valoriser, c'est-à-dire se donner de lui-même une "attitude positive " à son égard " (p. 148). Si chacun crée ses valeurs, c'est comme invitation à choisir de faire siennes des valeurs déjà déterminées ou objectives et, de ce fait, comme invitation à rencontrer l'autre. Cette rencontre responsable, objectivement et moralement bonne, réside dans le fait de l'appréciation mutuelle par l'intermédiaire des valeurs. Seuls les sujets libres peuvent valoir et être qualifiés de bons.

Examinant maintenant la contribution des valeurs et des obligations morales à la constitution d'une société où les droits et la liberté sont considérés comme fondamentaux, Lane remarque un droit général et originaire présidant aux autres : "celui de conduire soi-même sa propre vie, et pour le mieux » (p. 169). Pour donner cours à cette croyance et promouvoir la possibilité d'un bien commun, une condition est essentielle : celle du droit et de l'obligation universels et stables au respect. Ceux-ci sont " plus fondamentaux et plus originaires que tous les autres droits et obligations comme par exemple le droit à la vie elle-même, ou à tel ou tel bien-être, ou même au bonheur en général (. . .) » (p. 1.78).

La logique interne et factuelle des droits et obligations viserait donc l'établissement d'une société de semblables, sur une base commune d'attitudes et de comportements respectueux. En ce sens, tous et chacun devraient être prêts à n'importe quelle modalité concrète de l'échange, et ce en dépit des contrariétés et au prix d'une partie de leurs intérêts. Les souffrances pouvant de ce fait accompagner l'obligation absolue au respect ne pourront être compensées que par la confiance fondée sur la promesse initiale d'une rencontre fructueuse entre les semblables. Par la confiance, les uns et les autres s'obligent mutuellement à accomplir leur promesse de respect. Celleci est à la base socialisante et préside à la validité de toutes les lois positives. Cette loi du respect, soutient Lane, serait la seule à conférer l'obligativité aux contrats et aux lois positives. L'objection de conscience, la désobéissance civile, la révolution, le refus des minorités seront fort concevables à condition que les attitudes et les comportements des dissidents n'impliquent pas de manque de respect conscient à l'égard des autres. Sans le respect, la confiance serait privée de tout fondement. Tout bien-être ou bonheur ne peut être que la résultante de cette condition.

Car (chap. VIII) le bonheur de chacun semble dépendre de ce qu'il vaut et de ce qu'il fait valoir pour mériter respect et considération. Pour être 
heureux, il doit dépendre de nous que l'on vaille, que l'on puisse devenir une valeur "objective". Nous valons les uns auprès des autres. Ces autres qui nous sortent de l'accaparement des désirs et des craintes par l'aide d'un attrait que leur invitation rend efficace. Ces autres qui suscitent notre confiance dans un échange qui nous fait surgir à la fois de notre choix et du leur. Bref, il s'agit de valoir entre semblables à choisir la vie que l'on veut à la fois la meilleure et la plus heureuse.

Quant au plan politique maintenant, si nous devions transformer la fin des marionnettes ou de ce livre de Gilles Lane en souhait, nous aurions le programme suivant: que les dirigeants sociaux et politiques préparent et protègent les terrains propices à l'échange d'invitations et d'acceptations mutuellement libératrices entre les semblables, plutôt que de n'appliquer qu'un "social engineering" plus ou moins efficace à leur projet tout aussi factuel d'un problématique bien commun.

$\mathrm{Si}$, de ce souhait, il en va de l'animation des marionnettes, il en va tout autant de leur possibilité réelle de libre choix, celle-ci tenant dorénavant à leur existence et non plus à leur seule croyance. Libérées, les marionnettes seraient dès lors redevables à Gilles Lane d'avoir démontré que l'enjeu fondamental des droits de l'homme et des obligations morales afférentes est celui du respect. Le droit et l'obligation à celui-ci sont la condition de toute rencontre, de toute loi positive et de tout contrat. Ils fondent la confiance et la possibilité même de la liberté qui consiste à vouloir faire de soi-même et à valoir dans un advenir qui nous interpelle sans cesse par la médiation d'autrui. Redevables, les marionnettes le seraient, sauf à avoir préféré garder leur tête de bois.

Département de philosophie

Collège de Lévis-Lauzon 